\title{
Evolution of the leading-edge vortex over a flapping wing mechanism
}

\author{
M.R.Arifin' ${ }^{1}$, H. Yusoff' ${ }^{1}$ A.F.M. Yamin ${ }^{1}$, A.S. Abdullah'1 , M.F. Zakaryia², S. Shuib ${ }^{3}$ and S. Suhaimi ${ }^{3}$ \\ 1 Faculty of Mechanical Engineering, Universiti Teknologi MARA Cawangan Pulau Pinang, 13500, Permatang Pauh, Pulau Pinang \\ Phone: +6043823195 \\ 2 School of Mechanical Engineering, Universiti Sains Malaysia, Engineering Campus, 14300, Nibong Tebal, Pulau Pinang \\ ${ }^{3}$ Faculty of Mechanical Engineering, Universiti Teknologi MARA Shah Alam, 40450, Shah Alam, Selangor
}

ABSTRACT - Leading-edge vortex governs the aerodynamic force production of flapping wing flyers. The primary factor for lift enhancement is the leading-edge vortex (LEV) that allows for stall delay that is associated with unsteady fluid flow and thus generating extra lift during flapping flight. To access the effects of LEV to the aerodynamic performance of flapping wing, the threedimensional numerical analysis of flow solver (FLUENT) are fully applied to simulate the flow pattern. The time-averaged aerodynamic performance (i.e., lift and drag) based on the effect of the advance ratio to the unsteadiness of the flapping wing will result in the flow regime of the flapping wing to be divided into two-state, unsteady state $(\mathrm{J}<1)$ and quasi-steady-state $(\mathrm{J}>1)$. To access the benefits of aerodynamic to the flapping wing, both set of parameters of velocities $2 \mathrm{~m} / \mathrm{s}$ to $8 \mathrm{~m} / \mathrm{s}$ at a high flapping frequency of 3 to $9 \mathrm{~Hz}$ corresponding to three angles of attacks of $\alpha=0^{\circ}$ to $a=30^{\circ}$. The result shows that as the advance ratio increases the generated lift and generated decreases until advance ratio, $\mathrm{J}=3$ then the generated lift and drag does not change with increasing advance ratio. It is also found that the change of lift and drag with changing angle of attack changes with increasing advance ratio. At low advance ratio, the lift increase by $61 \%$ and the drag increase by $98 \%$ between $a=10^{0}$ and $a=200^{\circ}$. The lift increase by $28 \%$ and drag increase by $68 \%$ between $a=$ 200 and $a=30^{\circ}$. However, at high advance ratio, the lift increase by $59 \%$ and the drag increase by $80 \%$ between $a=100$ and $a=200$, while between $a=200$ and $a=300$ the lift increase by $20 \%$ and drag increase by $64 \%$. This suggest that the lift and drag slope decreases with increasing advance ratio. In this research, the results had shown that in the unsteady state flow, the LEV formation can be indicated during both strokes. The LEV is the main factor to the lift enhancement where it generated the lower suction of negative pressure. For unsteady state, the LEV was formed on the upper surface that increases the lift enhancement during downstroke while LEV was formed on the lower surface of the wing that generated the negative lift enhancement. The LEV seem to breakdown at the as the wing flap toward the ends on both strokes.
ARTICLE HISTORY

Revised: 21 st Apr 2020

Accepted: $28^{\text {th }}$ May 2020

\section{KEYWORDS}

Micro air vehicles; leading-edge vortex; aerodynamics.

\section{INTRODUCTION}

The American Defence Advanced Research Projects Agency (DARPA) defined Micro Air Vehicles (MAVs) as an aerial vehicle with a limited size less than $15 \mathrm{~cm}$. MAVs are also defined to have a flying speed of $10-20 \mathrm{~m} / \mathrm{s}$ or an operating Reynolds around 104-105. One of the challenges of MAVs comes from its small size and low Reynolds number operating condition that that have and adverse effect on the lift generation and aerodynamics performance. This is because according to traditional quasi-steady-state wing theory shows that it is difficult to generate lift during hover flight and slow flight speed [1].

However, lift generation for a small wing at low flight speed is not impossible because this phenomenon is common among nature's flyers and the secret for MAV design might lie in studying the forms found in nature. This act of looking at nature to solve a mechanical problem is often known as Bio-Inspiration or Bio-Mimicry design. Over the past several years, there have been several works that focuses on Bio-Inspiration design particularly on the mechanics and aerodynamics of flapping wings. The main motivation for Bio-Inspiration works can be divided into two major motivations; to understand the flight of animals and the designing a Micro Air Vehicles (MAVs) [2].

Although some progress has been made on the aeroelasticity of flapping wing especially in insect wing, most of the previous studies were conducted from a biologist's point of view to understand the fundamental mechanism and physics of flapping flights of natural flyers. As reported by Mueller et al. [3], there is a fundamental difference between an aerospace engineer's interest in flapping flight and that of a biologist or zoologist. For biologist or zoologist, the primary motivation for studying the flight mechanics of natural fliers is to explain the physics for a creature that can fly. On the other hand, an aerospace engineer is trying to develop a flying aircraft based on design-oriented analysis, which is not usually what can be offered from animal flight studies. Insects are able to hover by using a range of possible unsteady high-lift mechanisms, including rotational circulation [4,5], clap and fling [6,7], wake capture [8], and added mass $[9,10]$. 
However, arguably, the most important mechanism is LEV [11], which may generate up to two-thirds of the total lift in insect flight [12]. According to Maxworthy et al. [7], lift generation occurs in the unsteady and quasi-steady state regime and is hugely dependant on the strength of the leading-edge vortex (LEV) formed by the flapping motion. LEVs are also attributed the stall delay of the wing. The discovery of LEVs on the wings of insects in flight greatly advanced the knowledge of their dominant lift-generating mechanisms [13,14]. Sharp leading edges induce high lift production through flow separation with vertical flow attached to the upper surface of insect wings during flapping and gliding.

While there are a lot of works that studies LEVs, most of the works that have done focuses mainly on insects which is too small to be a basis for MAV design. A better base for MAV design should be flying vertebrates but slow flying vertebrates cannot be able to generate enough lift to maintain aloft. However, several species of small flying vertebrates are adapted to foraging using this flight mode. Species such as bats that flies well withing slow speeds during its foraging flight [15].

However, works done on the effects of LEVs formation on bat wings is still limited, therefore the purpose of this study is to study the effect of LEV and the effect of unsteady and quasi-steady flight regime on the aerodynamic performance of a bat wing.

\section{NUMERICAL METHODOLOGY}

\section{Governind Equation}

The governing equation for unsteady, incompressible fluid for the conservation equations of mass and momentum of the time-averaged continuity and RANS (Reynold averaged Navier-Stroke) equation is expressed as follows:

Continuity:

$$
\frac{\delta \bar{u}_{i}}{\delta x_{i}}=0
$$

Momentum (non-accelerating reference frame):

$$
\frac{\delta \bar{u}_{i}}{\delta t}+\frac{\partial}{\partial x_{j}}\left(\bar{u}_{j} \bar{u}_{i}\right)=-\frac{1}{\rho_{f}} \frac{\delta \bar{p}}{\delta x_{i}}+v \frac{\delta^{2} \bar{u}_{i}}{\partial x_{j}^{2}}
$$

where, $\rho_{\mathrm{f}}$ is the fluid density, $\mathrm{t}$ is the time, $\mathrm{p}$ is the pressure, $\mathrm{v}$ is the kinematic viscosity, and ui $(\mathrm{i}=1,2,3=\mathrm{u}, \mathrm{v}, \mathrm{w})$ and $\mathrm{u}_{\mathrm{j}}(\mathrm{j}=1,2,3=\mathrm{u}, \mathrm{v}, \mathrm{w})$ are the velocities in the $\mathrm{i}$-th and $\mathrm{j}$-th directions respectively. The directional tensors, $\mathrm{x}_{\mathrm{i}}$ and $\mathrm{x}_{\mathrm{j}}$, are defined for the $i$-th and $\mathrm{j}$-th directions $(\mathrm{i}=1,2,3=\mathrm{x}, \mathrm{y}, \mathrm{z}$ and $\mathrm{j}=1,2,3=\mathrm{x}, \mathrm{y}, \mathrm{z}$ ).

\section{Computational Methods and Boundary Conditions}

The numerical simulations of three-dimensional flow are performed using CFD package Ansys Fluent version 18. GAMBIT is used to generate the unstructured rectangular type grid of the computational fluid domain as shown in Figure 1. The figure also shows the model bat wing that is used for the study. The wing has a half wingspan of $124 \mathrm{~mm}$ and a chord length of $82 \mathrm{~mm}$. Unstructured tetrahedral cells are used owing to its three-dimensional dynamic moving grid skills for complex geometries like flapping wings [16]. The tetrahedral cells are more densely clustered near the wing to properly capture the critical flow details in these regions.

The non-slip boundary condition is employed at the wing wall. The velocity inlet and pressure outlet boundary conditions are used for inflow and outflow of the computational domain respectively. A slip boundary condition (symmetry) is specified on the tunnel walls to save computational cost and to avoid the need to resolve the boundary layer on these surfaces (Table 1).

Table 1. Ultimate tensile strength values and elongation to fracture.

\begin{tabular}{cc}
\hline Time & Transient \\
\hline Velocity Inlet $\left(\mathrm{ms}^{-1}\right)$ & $2,4,6$, and 8 \\
Pressure Outlet & Atmospheric \\
Walls & All walls: no slip \\
\hline
\end{tabular}

The domain is $9.7 \mathrm{~cm}$ in width and $4.9 \mathrm{~cm}$ in height. Uniform velocity profiles of $2 \mathrm{~m} / \mathrm{s}, 4 \mathrm{~m} / \mathrm{s}, 6 \mathrm{~m} / \mathrm{s}$ and $8 \mathrm{~m} / \mathrm{s}$ are used at the velocity inlet while a gauge pressure of zero is set at the pressure outlet. The unsteady, incompressible RANS equations are solved using an implicit, segregated, three-dimensional finite volume method. Temporal discretization is performed using an implicit, second-order scheme. A second-order accurate, upwind discretization scheme is used for 
momentum equations. A pressure-velocity coupling uses a SIMPLE algorithm to solve the resulting algebraic equation system. The turbulence viscosity is modelled by the Spalart-Allmaras (S-A) model. A grid independence study is performed and a suitable grid of 0.5 million cells is subsequently chosen.

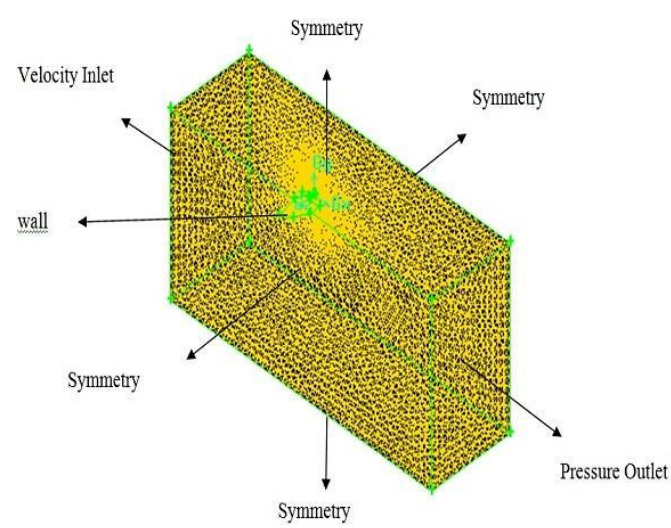

(a)

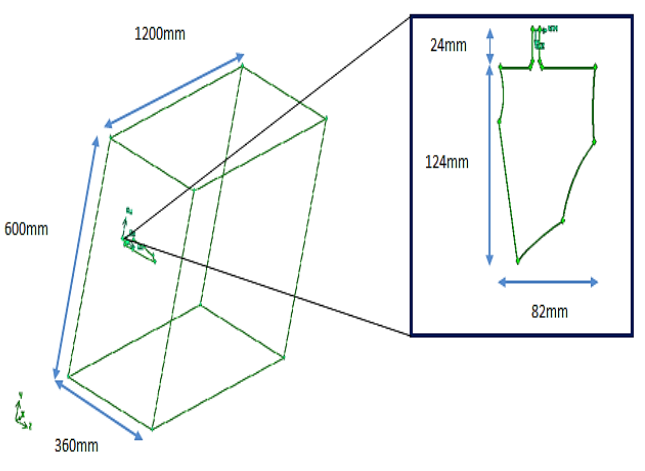

(b)

Figure 1. (a) Computational domain and (b) configuration of wing domain.

\section{RESULTS AND DISCUSSIONS}

\section{Unsteady Effects}

The non-dimensional parameter advance ratio, J, was used to characterise the unsteadiness of the flapping-wing flight. Advanced ratio, $\mathrm{J}$, which is defined as the ratio of forwarding flight speed or the freestream velocity to the wingtip velocity during flapping flight, can be expressed as:

$$
J=\frac{V_{\infty}}{2 b f \Phi} \max
$$

Where:

$\mathrm{J}$ is the advance ratio

$\mathrm{b}$ is the wing semi span

$\mathrm{f}$ is the wing flapping frequency

$\Phi_{\max }$ is the amplitude of wing flapping angle

$\mathrm{V} \infty$ is the freestream velocity.

Figure 2 and 3 shows the result of mean lift coefficients and mean lift drag coefficients respectively at difference advance ratio. It also shows results mean lift and drag coefficients at a different angle of attack (before stall angle). From Figure 2 and 3 show that at different conditions (different freestream velocity and flapping frequency) the result of the mean lift coefficients is found to align themselves nicely in the plots when the advance ratio, J, is used. It should also be noted that the relationships between the mean lift and drag coefficients and the advance ratio, J, can be represented well by exponential functions as stated in Figure 2 and 3 that are then fitted with the exponential decay functions. The exponential relationships between the mean lift and drag coefficients with the advance ratio of the flapping flight are also confirmed with the increment of mean lift coefficient at different angles of attack at the same advance ratio. The breakpoint between quasi-steady and unsteady flow is when $\mathrm{J}=1$. As stated by Ho et al., [2010], when $\mathrm{J}>1.0$, the flow around the flapping wing can be considered as quasi-steady, while $\mathrm{J}<1.0$ corresponding to the unsteady state regime. From the Figure 2, it can be noted that as the advance ratio become larger, the mean lift coefficients decrement is not significant indicating that it is in quasi-steady state which approaches a steady-state where lift coefficients are considered as constant.

As the advance ratio is reduced, the mean lift coefficients increment due to the effect of the unsteadiness is becoming more significant. The similar pattern also indicated the mean drag coefficients in Figure 3. From the formula of advance ratio as expressed previously, is a ratio of the freestream velocity to the wing tip velocity. A quasi-steady state, the flapping flight of the wing's flapping motion is at relatively low frequency (or hardly flapping at all) during the flight. Hence the wing tip speed is lower compared to the freestream velocity. These are examples of the larger birds such as eagles and seagulls, that usually considered to fly in quasi-steady regime since they usually flap they wing quite slowly that tend to have a soaring flight or level flight and their wings behave more like fixed wings. On the other hand, smaller birds such as hummingbird and insects fly in the unsteady state regime with their wings flapping at a much higher flapping frequency so the wingtip speed during flapping motion is much faster than the freestream velocity. Hence, when a flapping flight is in unsteady state regime, the flow moves around the flapping wing is considered highly unsteady and cannot 
easily be approximated by quasi-steady-state or steady-state assumptions. In this research, it can be seen that most of the flapping flight are in quasi-steady state regime $(\mathrm{J}>1)$ as in Figure 2.

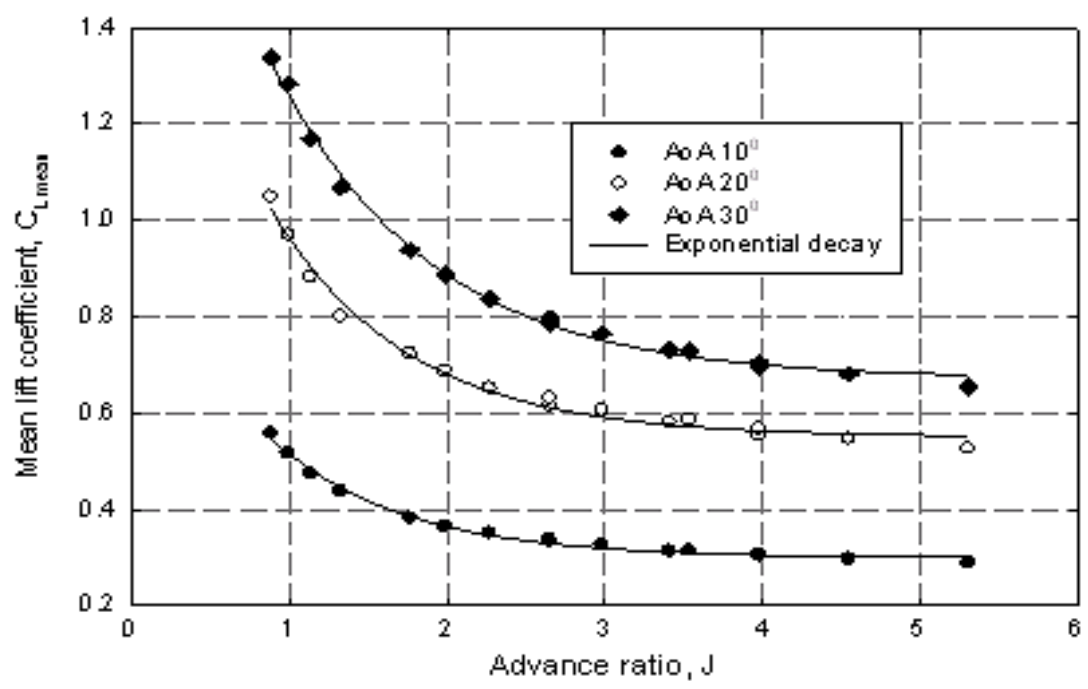

Figure 2. Mean lift conefficients versus advance ratio.

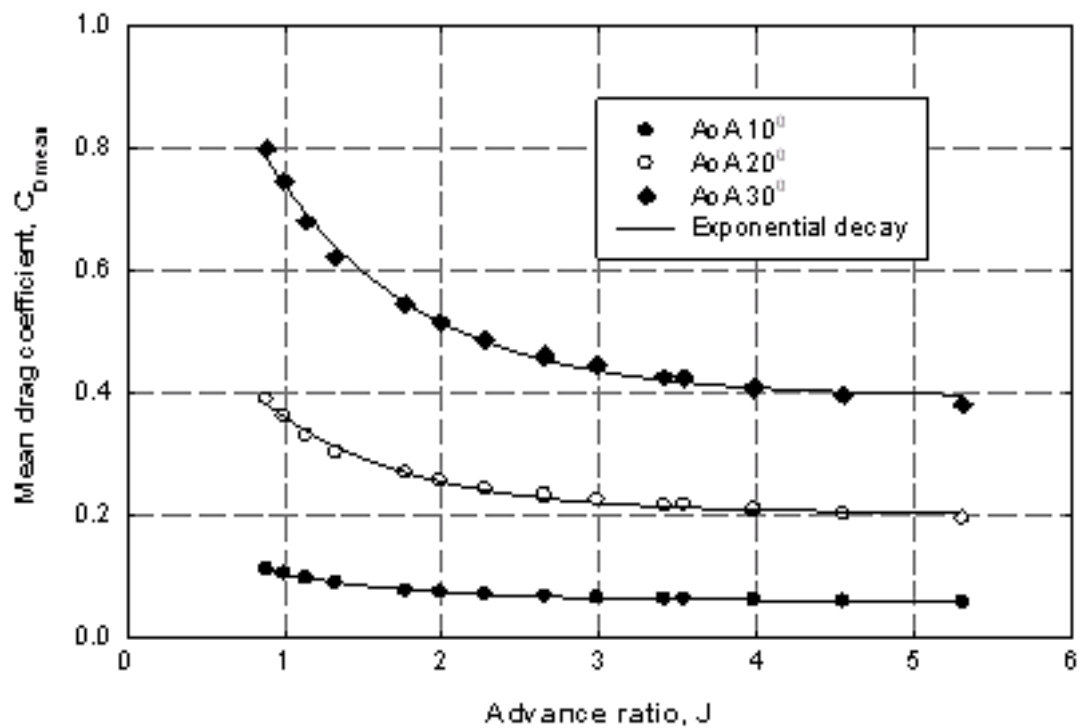

Figure 3. Mean drag coefficients versus angle of attack.

In terms of changing angle of attack, was found that at $\mathrm{J}=1$ the between $\alpha=10^{\circ}$ and $\alpha=20^{\circ}$, the lift increased by $61 \%$ and between angle of attack $30^{\circ}$ the lift increased by $28 \%$. At $\mathrm{J}=2$, the difference between $10^{\circ}$ angle of attack and $20^{\circ}$ is at $59 \%$ and the lift increases by $25 \%$ between $\alpha=20^{\circ}$ and $\alpha=30^{\circ}$. At later advance ratio, $(\mathrm{J}=4)$, it was found that the lift increases by $59 \%$ between $\alpha=10^{\circ}$ and $\alpha=20^{\circ}$, and the lift increases by $20 \%$ between $\alpha=20^{\circ}$ and $\alpha=30^{\circ}$. This shows that the lift slope of the wing started to decrease as the advance ratio increases. The same can be said about the generated drag where, at $\mathrm{J}=1$, the generated drag increases by $98 \%$ between $\alpha=10^{\circ}$ and $\alpha=20^{\circ}$ and the drag increases by $68 \%$ between $\alpha$ $=20^{\circ}$ and $\alpha=30^{\circ}$. As a higher advance ratio $(\mathrm{J}=2)$, the drag was found to increase by $87.5 \%$ between $\alpha=10^{\circ}$ and $\alpha=20^{\circ}$, and the drag increase by $67 \%$ between $\alpha=20^{\circ}$ and $\alpha=30^{\circ}$. At high advance ratio $(\mathrm{J}=4)$ it was found that the drag increase by $80 \%$ between $\alpha=10^{\circ}$ and $\alpha=20^{\circ}$, and the drag increases by $64 \%$ between $\alpha=20^{\circ}$ and $\alpha=30^{\circ}$. Again, this shows that the drag slope appears to decrease as the advance ratio increases because there is less increase of drag with increasing angle of attack as the advance ratio increase. 


\section{The Unsteady-State Flow Pattern}

From the previous discussion, the basic concept of the aerodynamic in unsteady and quasi-steady-state flow has been discussed. In this section, the detail of the flow on both regimes will be discussed and compared in more detail. To characterize the unsteady effect of the flapping flight, both quasi-steady and unsteady flow and has been discussed by choosing the right parameter on both states. For simplification, the same angle of attack of $10^{0}$ angles of attack at the same frequency is chosen. In this research, the velocity is chosen to study the unsteady effect rather than frequency. The detail is in Table 2. To understand the aerodynamic phenomena on the flapping wing, the detailed analysis must be investigated on both downstroke and upstroke. The critical phase of the analysis included early of the stroke, mid of stroke and the end of the stroke during both upstroke and downstroke. Six locations were considered at about those phases. For that, the three flapping angles are chosen, at about $\Phi \approx 30^{\circ},-30^{\circ}$ and $0^{\circ}$. The positions are as in Figure 4 .
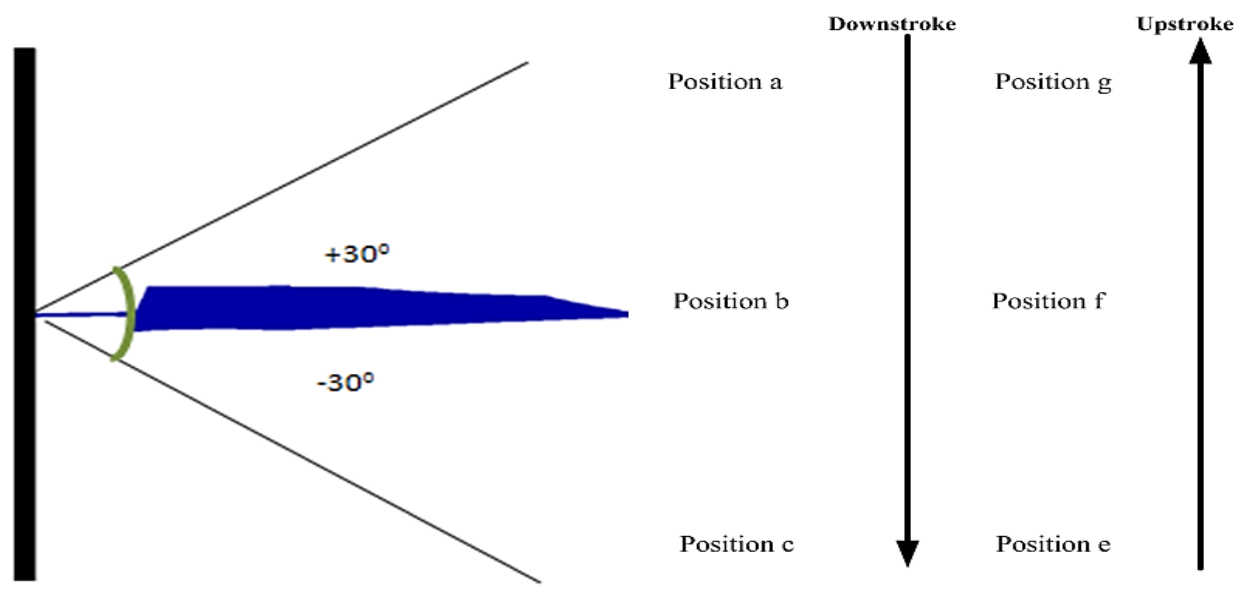

Figure 4. The position a, b, and c; at about early, mid and end downstroke respectively, for upstroke d, e and f about early, mid and end upstroke respectively with the total flapping angle equal of $60^{\circ}$.

Table 2. The different condition parameter at $\mathrm{AoA}=10^{0}$ for unsetady effect analysis.

\begin{tabular}{cccc}
\hline Flow regime & $\mathrm{V}\left(\mathrm{ms}^{-1}\right)$ & $\mathrm{f}(\mathrm{Hz})$ & $\mathrm{J}$ \\
\hline Unsteady & 2 & 9 & $0.8841076(\mathrm{~J}<1)$ \\
\hline
\end{tabular}

For the analysis, the condition such as pressure contour and velocity vector at the midplane $(\mathrm{b} / 2)$ and a plane about the tip (3b/4) where $b$ is the half of wingspan, has been chosen for analysis because it is the critical position for analysis the of the LEV formations. The LEV formation can be seen in Figure 5 using the path line where the direction and magnitude of the wind is shown with bluer arrows shows slower local wind speed, redder arrows shows faster local wing speed.

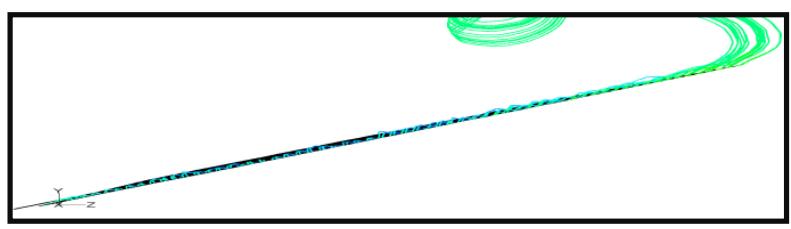

Position a

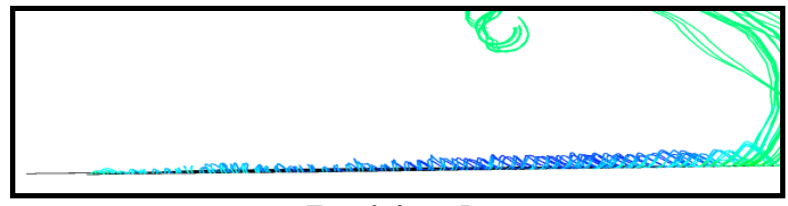

Position b

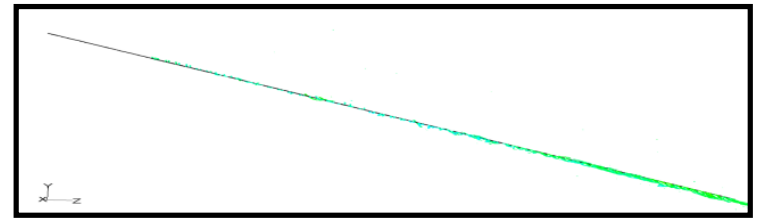

Position d

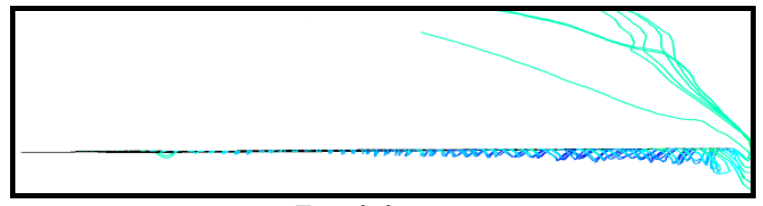

Position e 


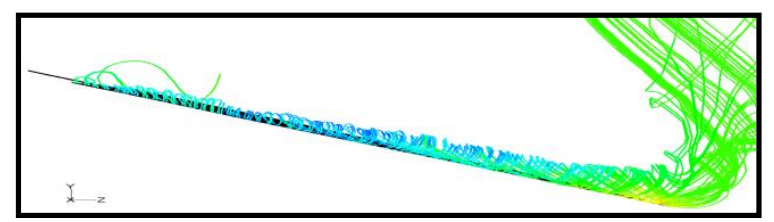

Position c

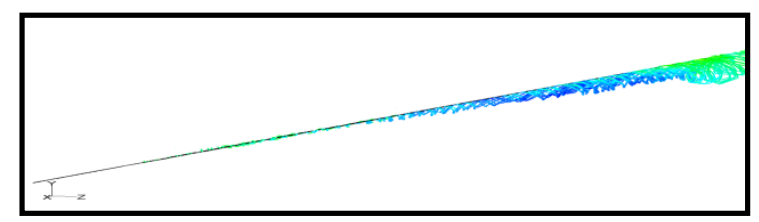

Position f

Figure 5. Pathline of pressure shows Leading Edge Vortex (LEV) formations over the flapping wing during the unsteady state.

From Figure 5, on the unsteady state, the LEV can be seen during downstroke on the upper surface and during the upstroke, the LEV can be seen on the lower surface. The LEV seems to be attached on the upper surface at the beginning (Figure 5a) and at the half of the downstroke (Figure 5c and Figure 5d) and detached at about the maximum downstroke (Figure 5d) where it is shed at during subsequent upstroke (Figure 5d).

The LEV can also be seen on the lower surface during upstroke. The similar pattern during upstroke can also be indicated on the lower surface. The LEV seems to be attached on the lower surface at the beginning (Figure 5d) and at the half of the upstroke (Figure 5e), LEV detaches at about the maximum position downstroke around the tip (Figure 5f). Figure 7(a) and 7(b) present the engineering stress-strain plots for 5052-6061 and 6061-5052, respectively. The purple curves represent the average values taken from three measurements, while the blue and red dashed lines indicate its $90 \%$ confidence levels (90\% CLs). Table 1 tabulates the ultimate tensile strength (UTS) and elongation values for both conditions. Overall, while the UTS is similar, it can be seen that the 6061-5052 joints exhibit greater consistency (based on a narrow $90 \%$ CLs) and a slightly better elongation to fracture, i.e. a tougher material property is achieved.

\section{CONCLUSIONS}

The effect of the advance ratio to the unsteadiness of the flapping wing will result in the flow regime of the flapping wing to be divided into two states, unsteady state $(\mathrm{J}<1)$ and quasi-steady state $(\mathrm{J}>1)$. It was shown that with increasing advance ratio the generated lift and drag decreases at all angles of attack until advance ratio, $\mathrm{J}=3$ where the generated lift and drag does not change with increasing advance ratio. It is also found that the lift and drag slope decreases with increasing advance ratio. This shows that wind speed influences the aerodynamic performance of the flapping wing. The way windspeed effects the generation of lift and drag can be seen by observing the unsteady state flow, the LEV formation during both strokes. The LEV is the main factor to the lift enhancement where it generated the lower suction of negative pressure. For unsteady state, the LEV was formed on the upper surface that increases the lift enhancement during downstroke while LEV was formed on the lower surface of the wing that generated the negative lift enhancement. The LEV seem to breakdown at the as the wing flap toward the ends on both strokes.

\section{ACKNOWLEDGMENTS}

The financial support from Universiti Teknologi MARA, Cawangan Pulau Pinang is highly appreciated.

\section{REFERENCES}

[1] D.J. Pines, F. Bohorquez "Challenges facing future micro-air-vehicle development," Journal of Aircraft 2006; 43:290-305

[2] Whitesides GM. "Bioinspiration: something for everyone," Interface Focus, vol.5, no.031, 2015.

[3] D. Mueller, H.A. Bruck, S.K. Gupta "Measurement of thrust and lift forces associated with drag of compliant flapping wing for micro air vehicles using a new test stand design," Experimental Mechanics, vol. 50, pp. 725-735, 2010.

[4] M. Jankauski, Z. Guo, I. Shen "The effect of structural deformation on flapping wing energetics," Journal of Sound and Vibration, vol. 429,pp. 176-192, 2018.

[5] M.H. Dickinson "The effects of wing rotation on unsteady aerodynamic performance at low Reynolds numbers," The Journal of Experimental Biology, vol. 192, pp 179- 206, 1994.

[6] T. Weis-Fogh "Quick estimates of flight fitness in hovering animals, including novel mechanisms for lift production," The Journal of Experimental Biology, vol. 59,pp. 169-230, 1973.

[7] T. Maxworthy "Experiments on the Weis-Fogh mechanism of lift generation by insects in hovering flight," Journal Fluid Mechanic, vol. 93, pp. 47-63, 1979

[8] R.B. Srygley, A.L.R. Thomas "Unconventional lift-generating mechanisms in free-flying butterflies," Nature, vol. 420, pp. 660-664, 2002. 
[9] C.P. Ellington "The aerodynamics of hovering insect flight. III. Kinematics," Phil Trans R Soc Lond B, vol. 305, pp. 41-78, 1984.

[10] S. Vogel "Life in Moving Fluids," Princeton Univ. Press, Princeton, NJ, 1994.

[11] C.P. Ellington, C. Van Den Berg, A.P. Willmott, A.L.R. Thomas "Leading-edge vortices in insect flight," Nature, vol. 384, pp. 626-630, 1996.

[12] C. Van Den Berg, C.P. Ellington "The vortex wake of a 'hovering' model hawkmoth," Phil Trans R Soc Lond B, vol. 352, pp. 317-328, 1997.

[13] R.J. Bomphrey, N.J. Lawson, N.J. Harding, G.K. Taylor, A.L.R. Thomas "The aerodynamics of manduca sexta: digital particle image velocimetry analysis of the leading-edge vortex," Journal of Experimental Biology, pp. 1079-1094, 2005.

[14] C. Gong, J. Han, Z. Yuan, Z. Fang, G. Chen "Numerical investigation of the effects of different parameters of three-dimensional flapping wings," Aerospace Science and Technology, vol. 84, pp. 431-445, 2019.

[15] E.K.V. Kalko "Insect pursuit, prey capture and echolocation in pipistrelle bats (microchiroptera)," Animal behaviour, vol. 50, pp. 861-880, 1995.

[16] Z.M. Fairuz, M.Z. Abdullah, H. Yusoff, M.K. Abdullah "Fluid-structure interaction of unsteady aerodynamics of flapping wing at low reynolds number," Engineering Applications of Computational Fluid Mechanics, vol. 7, no. 1, pp, 144-158, 2013. 